\title{
Electrical Characterization of Defects in Schottky Au-CdTe:Ga Diodes
}

\author{
P. Dyba, E. PŁaczek-Popko, E. Zielony, Z. Gumienny and J. Szatkowski \\ Institute of Physics, Wrocław University of Technology \\ Wybrzeże Wyspiańkiego 27, 50-370 Wrocław, Poland
}

Deep electron states in gallium doped CdTe have been studied by deep-level transient spectroscopy method. The Schottky $\mathrm{Au}-\mathrm{CdTe}$ diodes were processed to perform the investigations. Rectifying properties of diodes have been examined by the room temperature current-voltage and capacitance-voltage measurements. Deep-level transient spectroscopy measurements performed in the range of temperatures $77-350 \mathrm{~K}$ yield the presence of three electron traps. The thermal activation energies and apparent capture cross-sections have been determined from related Arrhenius plots. The dominant trap of activation energy $E_{2}=0.33 \mathrm{eV}$ and capture cross-section $\sigma_{2}=3 \times 10^{-15} \mathrm{~cm}^{2}$ has been assigned to the gallium related DX center present in the CdTe material.

PACS numbers: 81.05.Dz, 85.30.De, 84.37.+q

\section{Introduction}

It is well known that $\mathrm{Ga}$ forms in $\mathrm{Cd}_{1-x} \mathrm{Mn}_{x} \mathrm{Te}$ metastable defects, so-called DX centers, responsible for persistent photoeffects observed in these materials. The properties of the traps related to DX centers in $\mathrm{Cd}_{1-x} \mathrm{Mn}_{x}$ Te have been studied intensively (cf. [1] and references therein). According to the calculations carried out in [2] the shallow Ga state should be metastable with respect to the deep state also for Ga doped CdTe. Recently persistent photoeffects have been observed by us for gallium doped CdTe [3, 4] confirming this theory. However there are no reports on the DX-linked trap in this compound. In present paper in order to investigate the DX-related defect in Ga doped CdTe, deep-level transient spectroscopy (DLTS) method has been applied. The Schottky $\mathrm{Au}-\mathrm{CdTe}$ diodes have been processed. The rectifying properties of the diodes have been examined by the measurements of room temperature current-voltage $(I-V)$ and capacitance-voltage $(C-V)$ characteristics.

According to the thermionic emission model, the $I-V$ characteristics of a metal-semiconductor junction can be analyzed by the following equation [5]:

$$
\begin{gathered}
I=I_{0} \exp \left(\frac{q V}{n k T}\right)\left[1-\exp \left(-\frac{q V}{k T}\right)\right] \\
\text { with } I_{0}=S A^{*} T^{2} \exp \left(-\frac{q \Phi_{\mathrm{B}}}{k T}\right) .
\end{gathered}
$$

$I_{0}$ is the saturation current, $q$ - the electron charge, $n$ - ideality factor, $k$ - the Boltzmann constant, $T$ temperature, $S$ - the contact area and $\Phi_{\mathrm{B}}$ - Schottky barrier height. $A^{*}$ is the effective Richardson constant: $A^{*} \cong \frac{m *}{m_{0}} \frac{\mathrm{A}}{\mathrm{cm}^{2} \mathrm{~K}^{2}}$. For $\mathrm{CdTe} \frac{m *}{m_{0}} \cong 0.1$ resulting in $A^{*} \approx$ $12 \frac{\mathrm{A}}{\mathrm{cm}^{2} \mathrm{~K}^{2}}$. The saturation current can be determined by extrapolating the linear region of the forward $\ln I$ versus $V$ curve to zero voltage, for $V \geq 3 k T / q$ and then the Schottky barrier height can be deduced. The ideality factor of the diode can be calculated from the slope of the linear region.

For a metal- $n$-type semiconductor Schottky diode, $C-V$ measurements yield the donor net concentration $N_{\mathrm{D}}$ and the value of built-in voltage $V_{\mathrm{bi}}$. The relation between capacitance and voltage is given by [6]:

$$
\frac{S^{2}}{C^{2}}=\frac{2\left(V_{\mathrm{bi}}+V\right)}{\varepsilon_{\mathrm{s}} \varepsilon_{0} q N_{\mathrm{D}}},
$$

where $\varepsilon_{\mathrm{S}}$ is the semiconductor permittivity and $\varepsilon_{0}$ - the permittivity in vacuum. The slope of the $S^{2} / C^{2}$ against $V$ plot reveals $N_{\mathrm{D}}$ whereas $V_{\mathrm{bi}}$ can be obtained from the intersection between the $S^{2} / C^{2}$ line and voltage axis. The Schottky barrier height and built-in voltage are interrelated [6]:

$$
\Phi_{\mathrm{B}}=V_{\mathrm{bi}}+V_{n}+\frac{k T}{q}
$$

with $V_{n}$ - the distance between the bottom of conduction band and Fermi level given by following equations:

$$
\begin{aligned}
& V_{n}=\frac{k T}{q} \ln \frac{N_{\mathrm{C}}}{N_{\mathrm{D}}} \text { with } \\
& N_{\mathrm{C}}=4.83 \times 10^{15} T^{3 / 2}\left(\frac{m *}{m_{0}}\right)^{3 / 2} \mathrm{~cm}^{-3} .
\end{aligned}
$$

$N_{\mathrm{C}}$ is the effective density of states at the bottom of conduction band. Equations (3) and (4) allow to calculate $\Phi_{\mathrm{B}}$ once $N_{\mathrm{D}}$ and $V_{\mathrm{bi}}$ are known.

The dynamic process of capture and emission of electrons by majority deep traps in a Schottky metal- $n$-type semiconductor can be described in terms of a capture cross-section, $\sigma_{n}$, and emission rate, $e_{n}$. The electron 
emission rates are related to capture cross section by detailed balance equation [7]:

$$
e_{n}=\sigma_{n} \nu_{\mathrm{th}} N_{\mathrm{C}} \exp \left(-\frac{E_{\mathrm{T}}}{k T}\right),
$$

where $v_{\text {th }}$ is a thermal velocity of electrons and $E_{\mathrm{T}}$ is the apparent activation energy for transient emission. In the case of the DLS-82E system used in the DLTS experiment, the signal peak takes place at a temperature where lock-in frequency is related to emission rate by equation $e_{n}=2.17 f$. From Arrhenius plot i.e. the plot of experimentally obtained emission rates divided by the square of the temperature corresponding to the signal peak $\left(e_{n} / T^{2}\right)$ versus reciprocal temperature $(1 / T)$ apparent activation energy $E_{\mathrm{T}}$ and capture cross-section $\sigma_{n}$ of a trap can be determined. If the concentration $N_{\mathrm{T}}$ of the traps is much less than the donor concentration $N_{D}$, it can be found using relationship [7]:

$$
N_{\mathrm{T}}=2 N_{\mathrm{D}} \frac{\Delta C}{C},
$$

where $\Delta C / C$ is the relative change in capacitance corresponding to the signal peak amplitude.

\section{Experiment}

The samples of gallium doped CdTe were processed by the Bridgman method. The Schottky barriers were prepared by vacuum evaporation of gold $1 \mathrm{~mm}^{2}$ layer on front side of the CdTe slices chemically etched in a $2 \% \mathrm{Br}_{2}$ in methanol solution. Indium soldered to the backside of the slices served as an ohmic contact. $I-V$ measurements were performed with the constant current

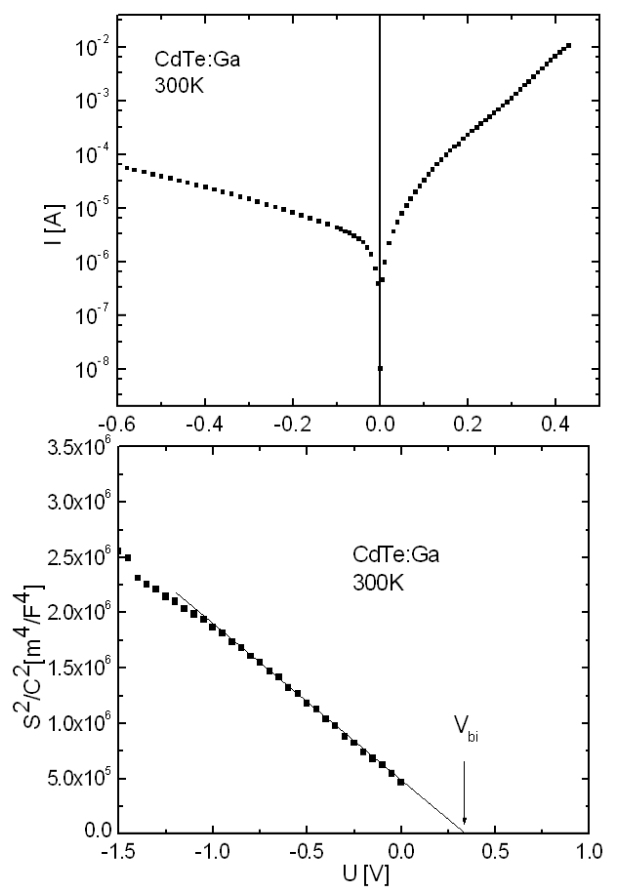

Fig. 1. Room temperature (a) $I-V$ and (b) $C-V$ characteristics.

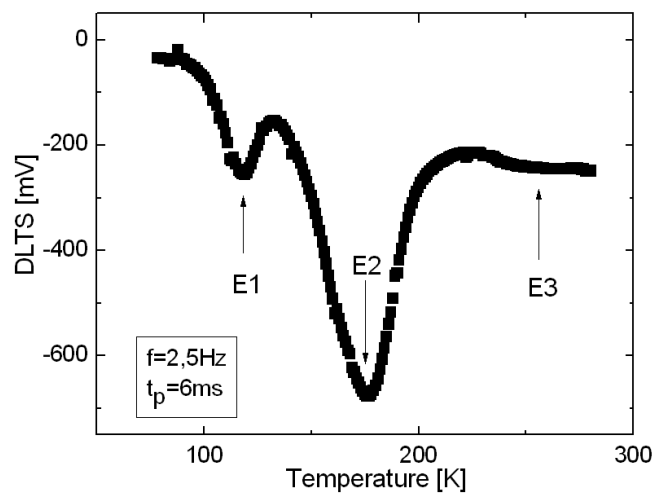

Fig. 2. Typical saturated DLTS spectra.

Keithley source, $C-V$ measurements - with Boonton 7200 capacitance bridge. DLTS measurements were done by DLS-82E System, SEMITRAP, Hungary.

Figure 1a shows a sample room temperature $I-V$ curve. The ideality factor and Schottky barrier height determined by Eqs. (1) and (2) were found to be equal to $n=2$ and $\Phi_{\mathrm{B}}=0.33 \mathrm{~V}$.

In Fig. 1b the room temperature $\frac{S^{2}}{C^{2}}-V$ characteristics is given. A good linearity of the characteristics up to $-1 \mathrm{~V}$ allowed to apply Eq. (3) and Eq. (4) to determine $V_{\mathrm{bi}}=0.33 \mathrm{~V}, N_{\mathrm{D}}=4.6 \times 10^{16} \mathrm{~cm}^{-3}, V_{n}=-0.05 \mathrm{~V}$ and $\Phi_{\mathrm{B}}=0.38 \mathrm{eV}$.

DLTS measurements were performed within the 77-350 K range of temperature and for different lock-in frequencies (in the range of $2.5 \mathrm{~Hz}-2.5 \mathrm{kHz}$ ). During the DLTS measurements the reverse bias was set at $1.5 \mathrm{~V}$ and periodically pulsed to $0 \mathrm{~V}$ for trap filling. Saturated capacitance transients were obtained with a pulse width of $6 \mathrm{~ms}$. A sample DLTS temperature scan measured at $2.5 \mathrm{~Hz}$ lock-in frequency is presented in Fig. 2.

Three peaks labeled as $E_{1}, E_{2}$ and $E_{3}$, related to majority electron traps can be noted. The concentration of the defects related to the dominant trap $E_{2}$ determined from Eq. (6) equals to $N_{\mathrm{T} 2} \approx 4 \times 10^{15} \mathrm{~cm}^{-3}$. DLTS

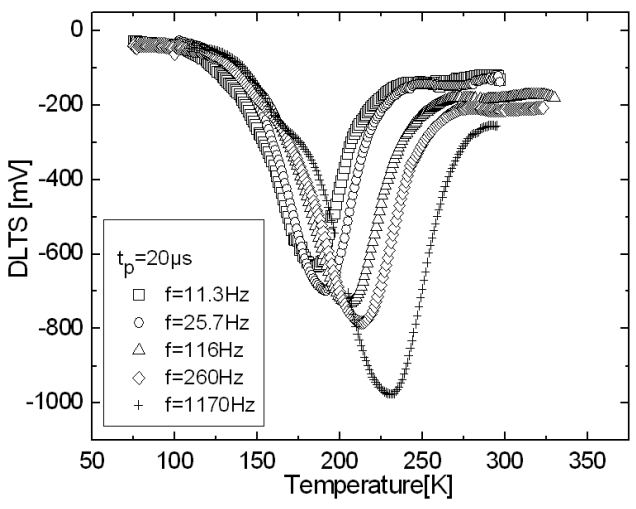

Fig. 3. DLTS spectra for different lock-in frequency. Reverse bias $1.5 \mathrm{~V}$, filling pulse height $1.5 \mathrm{~V}$ and its width $t_{\mathrm{p}}=20 \mu \mathrm{s}$. 


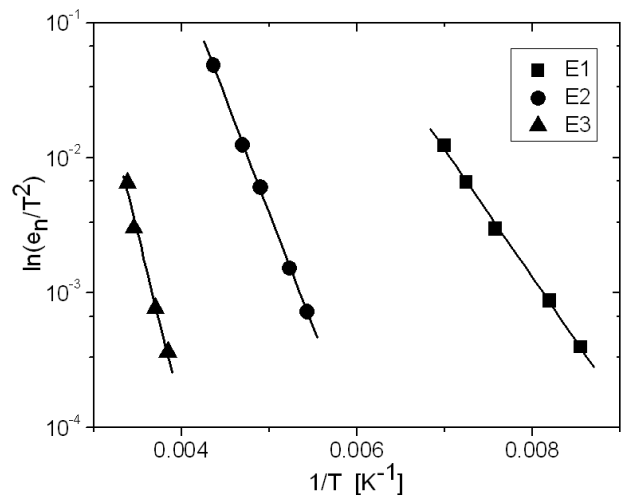

Fig. 4. Arrhenius plots for the traps $E_{1}, E_{2}$ and $E_{3}$.

spectra taken for different lock-in frequency are collected in Fig. 3. The Arrhenius data for the three levels are presented in Fig. 4. Apparent activation energies and capture cross-sections determined from the slope and intercept of a linear least squares fit to each set of data are summarized in Table. In Ref. [1] it has been shown that DX related DLTS signal predominates and its amplitude increases with increasing lock-in frequency, indicating thermally activated capture cross-section. Such a property exhibits the trap $E_{2}$. It is assumed that this trap is connected with gallium related DX centers in the studied CdTe.

TABLE

Apparent activation energy, capture cross-section and trap concentration.

\begin{tabular}{c|c|c|c}
\hline \hline Trap & $E_{\mathrm{T}}[\mathrm{eV}]$ & $\sigma\left[\mathrm{cm}^{2}\right]$ & $N_{\mathrm{T}}\left[\mathrm{cm}^{-3}\right]$ \\
\hline$E_{1}$ & 0.19 & $10^{-16}$ & $\approx 10^{15}$ \\
$E_{2}$ & 0.33 & $3 \times 10^{-15}$ & $4 \times 10^{15}$ \\
$E_{3}$ & 0.53 & $1.3 \times 10^{-14}$ & $\approx 10^{15}$
\end{tabular}

\section{Conclusions}

Fundamental electrical parameters of the Schottky $\mathrm{Au}-\mathrm{CdTe}$ diodes have been determined. Their rectifying properties have been examined by the room temperature $I-V$ and $C-V$ measurements. The ideality factor was found to be equal close to 2 and donor net concentration $N_{\mathrm{D}}=4.6 \times 10^{16} \mathrm{~cm}^{-3}$, the value of built-in voltage $V_{\mathrm{bi}}=0.33 \mathrm{~V}$ and the barrier height $\Phi_{\mathrm{b}} \approx 0.38 \mathrm{eV}$. DLTS measurements have been performed within the range of temperatures $77-350 \mathrm{~K}$. Three electron traps have been observed in the DLTS spectra. Apparent activation energies and capture cross-sections determined from related Arrhenius plots are following: $E_{1}=0.19 \mathrm{eV}$, $\sigma_{1}=10^{-16} \mathrm{~cm}^{2}, E_{2}=0.33 \mathrm{eV}, \sigma_{2}=3 \times 10^{-15} \mathrm{~cm}^{2}$, $E_{3}=0.53 \mathrm{eV}$ and $\sigma_{3}=1.3 \times 10^{-14} \mathrm{~cm}^{2}$. The trap $E_{2}$ is the predominant one. Its concentration equals to $N_{\mathrm{T} 2} \approx 4 \times 10^{15} \mathrm{~cm}^{-3}$ and it exceeds the concentrations of the other traps. This trap has been assigned to the gallium related DX center present in the CdTe material.

\section{Acknowledgments}

The work has been financially supported by the Ministry of Science and Higher Education project No. N N202 091133.

\section{References}

[1] E. Płaczek-Popko, J. Szatkowski, P. Becla, J. Appl. Phys. 95, 1171 (2004).

[2] C.H. Park, D.J. Chadi, Phys. Rev. B 52, 11884 (1995).

[3] E. Płaczek-Popko, Z. Gumienny, J. Trzmiel, J. Szatkowski, Opt. Appl. 38, 559 (2008).

[4] J. Trzmiel, E. Płaczek-Popko, K. Weron, J. Szatkowski, E. Wojtyna, Acta Phys. Pol. A 114, 1417 (2008).

[5] E.H. Rhoderic, R.H. Wiliams, Metal Semiconductor Contact, 2nd ed., Clarendon, Oxford 1988.

[6] S.M. Sze, Kwok K. Ng, Physics of Semiconductor Devices, 3rd ed., Wiley, Hoboken, New Jersey 2007.

[7] P. Blood, J.W. Orton, The Electrical Characterization of Semiconductors: Majority Carriers and Electron States, Academic Press, 1992. 